\title{
Body: A New Issue in Contemporary Calligraphy Aesthetics
}

\author{
JIANG Wen, ZHOU Wen-jie \\ Dalian University of Technology, Dalian, China
}

\begin{abstract}
With the development of new media, an important dimension of contemporary calligraphy has been highlighted, namely the body. One of the most intriguing phenomena of the body is the video of some calligraphy creations on the Internet. The exaggerated body movements and performances have caused heated discussion among the calligraphy circles and many netizens. However, the critics still lack deep and comprehensive thinking about the problems related to the body in calligraphy. We believe that the physical dimension of calligraphy refers to the appearance of the body in the practice of calligraphy in three ways: experience, imagery, and performance. Through the consideration of the body dimension of calligraphy, this article tries to put forward the general criticism of the calligraphy works and the creative practice of physical phenomena. It should be divided into the following parts. One of them is that this kind of physical phenomenon expresses the spirit of the times and expresses the individual "feeling"; "sorrow and joy" is helpful; the second is the merits and demerits of the work itself, and the third is the relationship between the generation of the work and the body and the degree of harmony.

Keywords: body, calligraphy, performance, aesthetics
\end{abstract}

\section{The Background of the Body Highlights: The Aesthetics of Calligraphy From the Essence of Beauty to Aesthetic Activities}

Calligraphic aesthetics is discussed earlier in China's academic circles, and its basics are compatible with the development of aesthetics in China. To put it simply, in the 1930s and 1950s, the introduction of Western aesthetics was basically over, and the older generation of estheticians such as Zong Baihua opened the modern path of Chinese calligraphy aesthetics into a system, a logic, and a utilitarian. In the 1960s and 1970s, calligraphy was influenced by the realist literary line at that time and the mainstream materialist ideology of mechanical materialism entered the period of silence. What followed was the golden period of calligraphy aesthetics in the 1980s. The calligraphy aesthetics discussion of this period brought people's understanding of calligraphy beauty to a new historical height. In the 1990s, Chinese calligraphy entered a period of reflection, focusing on reviewing and rethinking the discussion of calligraphy aesthetics. Later, scholars no longer revolved around the essence of calligraphy beauty. The study of calligraphy beauty by Jin Kaicheng, Qiu Zhenzhong, Zheng Xiaohua, Cong Wenjun, Wang Yuechuan, and other scholars showed the characteristics of language and culture. Then entering the 21st century, calligraphy aesthetics is no longer so popular.

JIANG Wen, Ph.D. student, Department of Philosophy, Dalian University of Technology, Dalian, China. ZHOU Wen-jie, Professor, Department of Philosophy, Dalian University of Technology, Dalian, China. 
Of course, in a few short sentences, it is difficult to describe in detail the development of Chinese calligraphy aesthetics for decades. However, from the approximate context, we can find that the contributions of these decades are obvious. First, the modern road of system, logic, and utilitarianism has become the direction of contemporary calligraphy aesthetic research efforts. This has basically become a consensus, laid a solid foundation for the construction of disciplines and the orderly development of calligraphy. Second, the great discussion on the essence of calligraphy beauty was carried out under the background of Chinese and Western aesthetics. This not only made calligraphy consolidate its independent aesthetic status, but also raised people's awareness of calligraphy beauty to a new height. However, we should also realize that the current research on calligraphy aesthetics still faces the problems of what to do after decades of development: First, the study of contemporary calligraphy theory, the historical materials test is flourishing. The study of calligraphy aesthetics has been neglected, and the study of calligraphy aesthetics has also shown the characteristics of cultural turn, that is, discussing the cultural connotation, cultural value, cultural significance, and so on of calligraphy in the broader context of culture. However, discussing calligraphy at the cultural level is not a substitute for calligraphy aesthetics. This has the possibility of immersing calligraphy in "nationalism". Therefore, we believe that calligraphy is not only the construction of oriental culture, but the artistic characteristics of calligraphy itself must be returned to the background of Chinese and Western aesthetics. Second, the discussion of the essence of the beauty of calligraphy is an extension of the aesthetic discussion of the nature of beauty. The great discussion of the essence of calligraphy is finally silenced in the reconciliation of practical aesthetics. Then, in addition to the essence of calligraphy, what else deserves further study and attention? We think it is an aesthetic activity. As early as the end of the 1980s, Jiang Peikun's “Analog of Aesthetic Activities” and Ye Lang’s “Modern Aesthetic System" had the characteristics of "from the basic research of ontology to the study based on aesthetic phenomena”. In fact, the basic research of aesthetic phenomena and ontology is the two wings of aesthetics—experience and logic. As Liu Chengji said,

As far as aesthetics is concerned, the object of concern should at least include two aspects: one is the question raised by life itself, which makes the aesthetic study always maintain the sense of the scene in sync with the times; the other is the problem derived from the inherent theory. This allows aesthetic studies to gain historical identity in the continuation of tradition. The former is empirical and the latter is logical. Mature theory often assigns empirical phenomena to logical forms and logical forms to establish order for experience. (Liu, 2005, p. 247)

Then, we can say that the essence of calligraphy beauty and the aesthetic activity of calligraphy are also such a relationship. The former focuses on speculation and tries to find a reliable logical starting point. This logical starting point must first be found in the body of calligraphy, so this stage is more beautiful for calligraphy. There is an expression, pictographic debate; the latter is concerned with the existence and development of aesthetic experience, that is, not only pay attention to the work, but also pay attention to the aesthetic experience of the subject, which provides the possibility for the appearance of the body in calligraphy aesthetics.

\section{The Appearance of the Body in Contemporary Calligraphy}

Once we turn our attention from the essence of calligraphy to the aesthetic activities of calligraphy, we will find that the body has developed itself in such activities. It can be said that the body is the most basic bearer of aesthetic activities in any art. 
The most direct aesthetic expression of the body is in various forms of art. Some art styles are themselves direct representations of the body, such as human sculptures and paintings; some art styles are also largely physical movements, such as dance, acrobatics, drama, singing, film, television, etc.; some art styles are neither direct expression of the body nor physical movements, but also describe and express the activities and feelings of the body, such as literature and music. In fact, any kind of art directly or indirectly relates to the human body. Without the depiction of the body, there is no art about human beings. An important characteristic of art is the revealing of the body itself. (Peng, 2005, p. 242)

So, from the body "Looking at art" has become a possible new topic in the aesthetics. This is different from the influence of Western philosophical philosophy in the past. It regards art as purely spiritual and discriminates the role of the body in art.

Body, this category is slightly stranger in calligraphy aesthetics, mainly because the body in calligraphy is concealed, and there is no deep discussion about the body in traditional calligraphy aesthetics (this is the overall integration of ancient Chinese mind and body). The body view is related; of course, this does not mean that the body is not important. Neglect of the body in calligraphy will lead us to aphasia about some of the physical problems in contemporary calligraphy. For example, with the development of the media, more and more self-media and platforms are free to publish videos written by calligraphers on the spot, as well as webcasting of calligraphy teaching. The rich paradigm that allows us to face the works from the past has become an appreciation paradigm for the entire creative process. This cannot but be said to be an improvement. This actually implies a change: The intentionality of calligraphy appreciation in the past is only a one-way reduction of the imaginary writing movement of the work. Now the appreciation of calligraphy can be written and played through video and live broadcast. The process of generation is presented at the same time. Such calligraphy can be described as more complete, making the entire aesthetic activity more lively, and the bearer of this sense of presence is nothing but the body. If the appearance of such a body is positive for calligraphy communication and teaching, then the following situation is much more complicated, that is, the sensational effect caused by exaggerated body movements in calligraphy creation in network communication. For example, some contemporary famous artists such as "Calling Calligraphy”, "Spray Ink Calligraphy”, "Blind Calligraphy”, and some exaggerated creation videos of "Jianghu Masters" have been on the Internet for a while. We know that these videos are not only in the calligraphy industry. Communication has also attracted the attention of many netizens. In fact, the attention of these netizens is more directed to the entertainment effects brought about by the exaggerated body movements in the creative process. So, how should calligraphy aesthetics judge this? Many of the current sounds judge their value and meaning by simply evaluating the work, such as categorizing their works into ugly calligraphy, and drawing good or bad conclusions. However, the conclusion that the vision is narrowly confined to the work is that it cannot explain and criticize the physical phenomena. Therefore, we believe that the evaluation of this phenomenon must open up the horizon, not only pay attention to the work but also pay attention to the body that has already appeared, and also make judgments on many aspects such as calligraphy aesthetics and artistic aesthetics, and can not help but think one-sidedly. Praise or slam, otherwise it will bring more misunderstandings and misleading. In addition, the body also appears in another face and deserves attention. Professor Wang Dongling of the China Academy of Art has introduced the aesthetic object of his body many times in his modern calligraphy. He wrote calligraphy on the picture of the human body album or in calligraphy. In this way, he hopes to establish a special relationship between the form of calligraphy and the form of the body. 
It can be seen that the appearance of the body in calligraphy is diverse. All of these kinds of methods are necessary for the body's attention and investigation. This is the field in which the practice has arrived, but the aesthetics of calligraphy is rarely involved. Therefore, the body is a new topic for contemporary calligraphy aesthetics.

\section{The Three Types of Body in Calligraphy Aesthetics}

In contemporary aesthetics, body aesthetics has become a manifestation. Many articles on body aesthetics have certain enlightening significance for examining the physical problems in contemporary calligraphy aesthetics. Among them, Fang Yingmin’s “What Is Body Aesthetics: A Critical and Developmental Study Based on the Definition of Body Aesthetics" argues that the concept of contemporary body aesthetics has at least three discourses or branches. The first is human aesthetics. Human aesthetics is mainly a discourse of aesthetic research on the external form of the body (mainly referred to as nude). The second is the study of the beauty of the consumer culture, that is, the contemporary masses use the means of material and technology to shape the aesthetics of the body in the induction and teaching of the consumer culture. Bodywork, beauty, plastics, clothing, fitness, advertising, and even violent, erotic physical practice fall into the category of this aesthetic. Finally, it is to cultivate and improve the body sensibility of body consciousness. This refers to Schusterman's definition of body aesthetics. "Schusterman's body sensibility is about the body's feelings, and it's about the body's inner perception and consciousness" (Fang, 2016, p. 18). Fang Yingmin's three concepts of body aesthetics actually involve two bodies. One is the appearance of the body; the other is the experience of the body. And there is often a tension between the two bodies, as Fang Yingmin said, "For the appearance and experience of the actual individual body, it is realistic and possible to see that the unity of the two is greater than the opposite. Otherwise, he either means the division of the individual itself or is morbid". "Overall ( Extreme, case cases) In the actual body aesthetic practice, the appearance and experience of the individual body are unified, which is a basic aesthetic fact” (Fang, 2016, p. 21). Based on this consideration, Fang Yingmin tried to unify these three discourses and the two bodies through a more comprehensive concept and theoretical system. After argumentation and combing, it is believed that the so-called body aesthetics is the aesthetic practice of human beings centering on the shaping, appreciation, and display of body beauty.

Fang Yingmin's contribution is obvious, and the definition of his body aesthetics will correct such mistakes to a certain extent-seeing the appearance or experience of the body, one of which is considered to be the whole body aesthetics. On the other hand, starting from the aesthetic practice, it is possible to unify the experience and appearance of the body, so that the organic aesthetics is within the body aesthetics. However, such a theoretical system (body beauty shaping theory, body beauty appreciation theory, body beauty display theory) cannot be directly applied to calligraphy, because body aesthetics must be built around the beauty of the body. The center of calligraphy is to show the beauty of art rather than the beauty of the body. What we want to examine is the role that the body bears in the practice of calligraphy aesthetics. But this does not mean that the system of Fang Wen is useless for the theoretical system construction of the body dimension of calligraphy. We can learn from this structure to conduct a study of calligraphy around the body. First of all, from the appreciation of body beauty in calligraphy, the appreciation of calligraphy and the appreciation of body shape seem to have a different world. However, 'the way in which text can be created can only be the posture of 'viewing' or 'birds and beasts' when 
'seeing and looking up'. 'Looking at the view', this far-reaching wording posture, right calligraphy has a key influence” (Qiu, 2016, p. 78). It can be seen that, because of the creation of calligraphy or words, the way of "getting close to the body and taking things away" has already implanted the image of the body (shape, rhythm, function, etc.) into the deepest of calligraphy and Chinese characters. In addition, for people, the body is the most familiar object of the ancestors, not only familiar with the appearance of the body, but also more familiar with the functions of various body parts. Therefore, the body pushes the natural and the universe, and the mountain has a mountain pass, a mountainside, a mountain ridge, and the pot has a spout, a body, and the like. At the same time, people in the Han Dynasty gradually realized the difference between the human body and the shape and internal character of the human being, which made the bone phase method prosper and penetrated into the daily life of the people and the official talents, in the recommendation system. Thus, the richer understanding of the body makes the paradigm of the body enter the art field, and calligraphy is no exception. In the Wei and Jin Dynasties, the evaluation of calligraphy, fat and thin bones, has become an important evaluation standard. Therefore, the appreciation of calligraphy actually includes appreciation of the symmetry and harmony of the body image. Because of this, this type of body dimension in calligraphy can be called body image theory. Secondly, Fang Yingmin's so-called body beauty shaping theory is experiential theory. On the one hand, it refers to the shaping of the body shape; on the other hand, it refers to the shaping of body consciousness. The former generally refers to the traditional cultural background of learning calligraphy on the body of the United States to supplement the role. More importantly, the latter refers to the shaping of the body consciousness as "the body of feeling", where the body assumes the role of the subject of experience. The body participates in the construction of calligraphy beauty, which makes calligraphy beauty base on the most realistic and emotional physical experience. Of course, the calligrapher's experience is more important, as well as the writing experience of "the time of luck". Therefore, this type of body dimension of calligraphy is called experience theory. Finally, it is the theory of representation, which points to the body as a sensuous activity, which includes body physique in daily life and body performance in art in body aesthetics. Obviously, calligraphy is not the art that the body needs to play. But this does not mean that the body is insignificant in the role of calligraphy. The reason why calligraphy has such rich cultural connotations and foundations is that the important reason is the participation of the body.

The glyph should adapt to the physiological conditions of human writing, mainly the movement physiology of the hand. It is suitable for the writing conditions of the hand, it is smooth to write, it is quick and beautiful. Because of this, people are transforming according to the physiological requirements of writing. From the original pictorial characters, the glyphs are constantly being transformed under the physiological requirements of writing. It is under such requirements that the words are constantly changed from lines depicting objective foreign objects to typed lines that are convenient to write; Constantly changing from a curve to a straight line, and even turning a straight line into a streamline, this transformation process has become more and more easy to change the appearance of the word. Chinese characters are made up of big and small, and are written by scripts, from Lishu and script, and from Lishu and Shu. The scripts and cursive scripts are all written and written, and are determined partly or mainly by the writing physiology. (Wang, 2018, p. 37)

It can be seen that the writing physiology is to follow the prescriptive nature of the physical reality (natural body), and the practice of calligraphy techniques is the activity of transcending the writing physiology, that is, the construction of the cultural body, the tension between the two is that the history of calligraphy has an important significance. Therefore, the body with cultural and natural duality as a perceptual activity and its expressive 
movements have contributed to the rich expression and cultural connotation of giving calligraphy. Therefore, the body dimension we are talking about here points to the expressive movements in calligraphy practice. For this type of body dimension, we call it expression theory.

In summary, physical problems play an important role in calligraphy. Crossing or ignoring the physical problems in calligraphy will make calligraphy one-sided: The way of existence or the complete aesthetic activity is completely narrowly equivalent to the appreciation of calligraphy works, and the aesthetic type is completely limited to artistic beauty. This leads to aphasia to some of the problems in calligraphy. Therefore, paying attention to the body dimension of calligraphy is meaningful, and it is more necessary to clarify what it refers to. Therefore, we believe that the body dimension of calligraphy refers to the appearance of the body in the practice of calligraphy in three ways: experience, imagery, and performance.

\section{Reflection on a Physical Phenomenon in Contemporary Calligraphy}

Through the above brief analysis, we can know the different roles that the body bears in the art of calligraphy. The theory of experience, imagery, and expression can comprehensively summarize the physical phenomena in calligraphy. We can perceive that with the development of contemporary calligraphy art and new media, the dimensions of the body in calligraphy have gradually emerged, especially in the body of expression theory. This body points to the body that appears in calligraphy creation in the contemporary era. The body movements are synchronized with the generation of calligraphy works. The exaggerated movements and screams of the body become part of the process of creation. This kind of physical phenomenon is very eye-catching and has a certain typicality in the contemporary. Therefore, we will analyze this phenomenon and try to establish the role and status of this body phenomenon in the process of calligraphy generation, and thus judge its own meaning.

In reading this phenomenon, many critics often go to Zhang Xu and Huai Su to find legitimacy. We know that the performance of Zhang Xu and Huai Su's calligraphy once attracted the praise of many poets at that time, but they have also been criticized by Su Shi and accused of having the elements of dazzling skills. This is because the performance of this kind of body is brought about by the coupling of many times, and the background of that era will appear abrupt and artificial.

At that time, the great poets such as Gao Shi, Li Bai, Du Fu and Zhang Xu had contacts, except for the performances of the writers such as Zhang Xu and Huai Su, and more importantly, their artistic style promoted the spirit of the times. Poets also pay attention to a time proposition. (Gan, 2016, p. 162)

The performance of the performing body in the Tang Dynasty was endowed by the spirit of the times. This period is the historical stage in which the "emotionality" of human beings and the beauty of calligraphy are united. Before Zhang Xuhuai, the writers eagerly hoped to find more madness. The free form of calligraphy communicates to reveal and express "emotionality", and this ideal and goal has finally been reached in Zhang $\mathrm{Xu}$ and Huai Su, and the performance of the body has become a channel for communication of "emotionality" and calligraphy. This kind of performance is the result of the externalization of "emotionality", and this "feeling" is obtained in this kind of expressive action, and further injected into the madness, novelty, and teacher-centered in the wild grass. It can be seen that the performance of this body is inspired by a combination of factors. Therefore, as the Song people injected the literati's emotional experience, values, and world outlook 
into calligraphy, the cultural connotation of calligraphy greatly increased. The Song people no longer satisfied the value of seeking calligraphy in the form, but went outside the form to find the pursuit of rhyme, meaning, interest, and sloppiness is the portrayal of the aesthetics of the writers in this period, which makes the external form of physical performance dwarf, thus shaking the foundation of its existence. This explains reasonably why Su Shi and Mi Fu should face the swearword of Zhang Diansu; on the other hand, the gaze shifts from the background of the times to the practice of individual creation. When it comes to the expressive movements of calligraphy, we often refer to Jiang Baishi. A paragraph: "When I was reading ancient calligraphy works, there was no movement without drawing strokes, as if I saw the author's writing”. It can be seen that an excellent calligraphy work can be restored to expressive action in an appreciative appreciator, which undoubtedly gives us some inspiration: In the calligraphy tradition, the legitimacy of physical performance is conditional, that is, the body. The expression is highly harmonious with the works. If the two are not harmonious, then the performance of the body is superfluous and worthless; and the calligraphy works between the two are the dominant forces, and then the wonderful body. The final result of the performance is a poor work, and this creation is undoubtedly a failure. Too much emphasis on the body and neglect of the work or the discord between the body and the work can lead to the derogation of the value of the calligraphy itself. Therefore, when looking at the phenomenon of physical performance in contemporary calligraphy, we can at least get two principles from the tradition: First, from the boundary, calligraphy must be written with a brush; second, from the hierarchical sequence, In such artistic activities, calligraphy work is the most important, and the physical performance cannot surpass the work and become the main aesthetic object. In the following, the overall criticism of the calligraphy works and the creative practice of the body can be divided into the following parts. One of them is whether this kind of physical performance can help express the spirit of the times and express personal "emotion". The second is the pros and cons of the work itself, and the third is the relationship between the generation of the work and the performance of the body and the degree of harmony.

Then, it is difficult to get the support of traditional calligraphy aesthetics by using non-brushed tools for so-called "writing" or writing non-Chinese characters with a brush, and even for the artistic practice of highlighting the value of the calligraphy works by highlighting the performance of the body. Although such a phenomenon cannot find its legal basis in calligraphy aesthetics, it still has the possibility of legitimacy in the large field of art. This legitimacy comes not only from the free nature of aesthetics but also from the individual of the artist, the achievement of aesthetic ideals. Therefore, for the appearance of various kinds of bodies in contemporary calligraphy, we should use the above theory to judge which are the legality of traditional calligraphy and which break the legitimacy of calligraphy, and break through the "performance" of the legitimacy of calligraphy. It can be seen as a "behavioral art" using the form of calligraphy creation. Through the above judgments and critical principles, we will defend the core values and traditions of calligraphy art, and guard the boundaries and bottom line of calligraphy.

\section{References}

Fang, Y. M. (2016). What is body aesthetics—A critical and developmental investigation based on the definition of body aesthetics. Journal of Guizhou University (Social Science Edition), (1), 16-25.

Gan, Z. L. (2016). History of Chinese calligraphy criticism. Beijing: People’s Fine Arts Publishing House.

Liu, C. J. (2005). A contemporary case of body aesthetics. Zhongzhou Academic Journal, (3), 247-248. 
Peng, F. C. (2005). Basic problems in body aesthetics. Zhongzhou Academic Journal, (3), 241-244.

Qiu, X. Q. (2016). Poetics of posture: The origin of daily writing and calligraphy. Hangzhou: Zhejiang People’s Fine Arts Publishing House.

Wang, F. Y. (2018). Chinese characters (Volume 1). Beijing: Zhonghua Book Company. 